Article

\title{
Large Time Behavior for Inhomogeneous Damped Wave Equations with Nonlinear Memory
}

\author{
Mohamed Jleli ${ }^{1} \mathbb{D}$, Bessem Samet ${ }^{1}$ and Calogero Vetro ${ }^{2, *(\mathbb{D}}$ \\ 1 Department of Mathematics, College of Science, King Saud University, P.O. Box 2455, Riyadh 11451, \\ Saudi Arabia; jleli@ksu.edu.sa (M.J.); bsamet@ksu.edu.sa (B.S.) \\ 2 Department of Mathematics and Computer Science, University of Palermo, Via Archirafi 34, \\ 90123 Palermo, Italy \\ * Correspondence: calogero.vetro@unipa.it
}

Received: 3 September 2020; Accepted: 23 September 2020; Published: 27 September 2020

\begin{abstract}
We investigate the large time behavior for the inhomogeneous damped wave equation with nonlinear memory $\phi_{t t}(t, \omega)-\Delta \phi(t, \omega)+\phi_{t}(t, \omega)=\frac{1}{\Gamma(1-\rho)} \int_{0}^{t}(t-\sigma)^{-\rho}|\phi(\sigma, \omega)|^{q} d \sigma+\mu(\omega), t>0$, $\omega \in \mathbb{R}^{N}$ imposing the condition $\left(\phi(0, \omega), \phi_{t}(0, \omega)\right)=\left(\phi_{0}(\omega), \phi_{1}(\omega)\right)$ in $\mathbb{R}^{N}$, where $N \geq 1, q>1$, $0<\rho<1, \phi_{i} \in L_{l o c}^{1}\left(\mathbb{R}^{N}\right), i=0,1, \mu \in L_{l o c}^{1}\left(\mathbb{R}^{N}\right)$ and $\mu \not \equiv 0$. Namely, it is shown that, if $\phi_{0}, \phi_{1} \geq 0$, $\mu \in L^{1}\left(\mathbb{R}^{N}\right)$ and $\int_{\mathbb{R}^{N}} \mu(\omega) d \omega>0$, then for all $q>1$, the considered problem has no global weak solution.
\end{abstract}

Keywords: damped wave equation; inhomogeneous term; nonlinear memory; global weak solution; nonexistence result

MSC: 35L05; 35B44; 35B33

\section{Introduction}

We study the Cauchy problem of nonlinear damped wave equation in the form

$$
\phi_{t t}-\Delta \phi+\phi_{t}=\frac{1}{\Gamma(1-\rho)} \int_{0}^{t}(t-\sigma)^{-\rho}|\phi(\sigma, \omega)|^{q} d \sigma+\mu(\omega) \quad \text { in }(0, \infty) \times \mathbb{R}^{N},
$$

imposing the initial condition

$$
\left(\phi(0, \omega), \phi_{t}(0, \omega)\right)=\left(\phi_{0}(\omega), \phi_{1}(\omega)\right) \text { in } \mathbb{R}^{N},
$$

where $N \geq 1,0<\rho<1, q>1, \phi_{i} \in L_{\text {loc }}^{1}\left(\mathbb{R}^{N}\right)$ with $i=0,1, \mu \in L_{\text {loc }}^{1}\left(\mathbb{R}^{N}\right)$ and $\mu \not \equiv 0$. The integral in the right-hand side of Equation (1) is known as "nonlinear memory".

We recall that the choices of both the domain and boundary conditions may influence significantly the properties and behavior of the physical system, which is mathematically represented by the above Equation (1). In general, the wave type equations are fundamental tools in recasting various propagation phenomena and in developing methods for numerical solving the physics problems. In detail, symmetries of wave type equations and their solutions have been pointed out and investigated by many contributors. We mention that the symmetry's properties were successfully used to obtain ortogonality's criteria for the existence of solutions in elastic and anisotropic media (see, for example, the pioneering papers of Love [1], Woodhouse [2], Chapman-Woodhouse [3], and the references therein). About the computational approach to the study of wave type equations, we recall that the nonlinear wave equations can be linked to linear wave equations, using symmetry transformations (in particular, 
non-local transformations). So, we can find a correspondence one-to-one between the solutions of nonlinear and linear wave equations. Resuming, each nonlinear wave equation can be linearized by a non-local symmetry analysis (for more details, see Bluman-Cheviakov [4]). For further discussion about the potential benefit of this procedure, we refer to the papers by Taylor-Kidder-Teukolsky [5] and Palacz [6] (spectral methods for propagation phenomena).

Here, we ask the question of whether the problem (1) and (2) admits global weak solutions. The interest in such a kind of results is motivated by a wide literature on what can be called "large time behavior of solutions" to wave type problems, which consists in providing sufficient criteria to the existence, nonexistence and blow-up of solutions to some classes of parabolic differential equations. For this purpose, we employ the test function method.

Now, we recall some important results related to the blow-up of solutions to damped wave equations. First, we refer to the semilinear damped wave equation

$$
\phi_{t t}-\Delta \phi+\phi_{t}=|\phi|^{q} \quad \text { in }(0, \infty) \times \mathbb{R}^{N}
$$

imposing (2). In the work of Todorova-Yordanov [7], can be found the following results:

- If $q \in\left(1,1+\frac{2}{N}\right)$ and $\int_{\mathbb{R}^{N}} \phi_{i}(\omega) d \omega>0, i=0,1$, then there is no global solution to problem (2) and (3);

- If $q \in\left(1+\frac{2}{N}, \frac{N}{N-2}\right)$ for $N \geq 3$, and $q \in\left(1+\frac{2}{N}, \infty\right)$ for $N \in\{1,2\}$, then a unique global solution exists, under suitable initial values.

In the literature, the exponent " $1+\frac{2}{N}$ " is known as the critical Fujita exponent. Indeed, it is critical for the problem (2) and (3), but it is also the critical exponent for the semilinear heat equation (see Fujita [8])

$$
\phi_{t}-\Delta \phi=|\phi|^{q} \quad \text { in }(0, \infty) \times \mathbb{R}^{N} .
$$

Further studies in Kirane-Qafsaoui [9] (semi-linear wave equation with linear damping) and Zhang [10] (nonlinear wave equation with damping), established that $1+\frac{2}{N}$ belongs to the nonexistence case.

It is worth pointing out that in the limit case $\rho \rightarrow 1^{-}$, (1) reduces to

$$
\phi_{t t}-\Delta \phi+\phi_{t}=|\phi|^{q}+\mu(\omega) \text { in }(0, \infty) \times \mathbb{R}^{N} .
$$

The above equation was recently investigated by Jleli-Samet [11], who established the following results:

- If $\mu \in L_{l o c}^{1}\left(\mathbb{R}^{N}\right), \mu \geq 0, \mu \not \equiv 0, q \in\left(1,1+\frac{2}{N-2}\right)$ for $N \geq 3$, and $q \in(1, \infty)$ for $N \in\{1,2\}$, then there is no global weak solution to (4);

- If $N \geq 3$ and $q \in\left(1+\frac{2}{N-2}, \infty\right)$, then global solutions exist for suitable $\mu>0$.

This means that the critical exponent for Equation (4) is given by

$$
q_{c}(N)=\infty, \quad N \in\{1,2\}
$$

and

$$
q_{c}(N)=1+\frac{2}{N-2}, \quad N \geq 3 .
$$

Remark 1. From the above result, one observes the considerable effect of the inhomogeneous term $\mu$ on the critical behavior of (2) and (3). Namely, for $N \geq 3$, the critical exponent for (4) jumps from $1+\frac{2}{N}$ (the critical exponent for (2) and (3)) to the bigger exponent $1+\frac{2}{N-2}$. Notice that a similar phenomenon was observed for the heat equation [12] (Zhang, 1998) and the wave equation [13] (Zhang, 2000). 
An interesting wave type problem is driven by the equation:

$$
\phi_{t t}-\Delta \phi+\phi_{t}=\int_{0}^{t}(t-\sigma)^{-\rho}|\phi(\sigma, \omega)|^{q} d \sigma \quad \text { in }(0, \infty) \times \mathbb{R}^{N},
$$

assuming the initial condition (2). Problem (2) and (5) was first investigated by Fino [14], who proved the results as follows. Let

$$
q(N, \rho)=\max \left\{q_{\rho}(N), \rho^{-1}\right\},
$$

where

$$
q_{\rho}(N)=1+\frac{2(2-\rho)}{[N-2(1-\rho)]_{+}} .
$$

Thus, we have:

- If $N \in\{1,2\}$, then $q(N, \rho)=q_{\rho}(N)$, for all $\rho \in(0,1)$;

- If $N \geq 3$, then

$$
q(N, \rho)=\left\{\begin{array}{lll}
q_{\rho}(N) & \text { if } \quad \frac{N-2}{N} \leq \rho<1 \\
\rho^{-1} & \text { if } \quad 0<\rho \leq \frac{N-2}{N} .
\end{array}\right.
$$

Moreover, a finite time blow-up occurs in the following cases (see again [14]):

- If $q \in\left(1, q_{\rho}(N)\right]$ and $q(N, \rho)=q_{\rho}(N)$, i.e., for all $\rho \in(0,1)$ when $N \in\{1,2\}$, or for all $\rho \in\left[\frac{N-2}{N}, 1\right)$ when $N \geq 3$;

- $\quad$ If $q \in\left(1, \frac{N}{N-2}\right]$ and $q(N, \rho)=\rho^{-1}$, i.e., $N \geq 3$ and $\rho \in\left(0, \frac{N-2}{N}\right]$.

Finally, by considering compactly supported functions $\left(\phi_{0}, \phi_{1}\right) \in H^{1} \times L^{2}$ with small values, in [14] a global existence result is derived in the case $N \leq 3$ and $q>q_{F}(N, \rho)$, where

$$
q_{F}(N, \rho)=\left\{\begin{array}{ccc}
1+\frac{2(3-2 \rho)}{2 \rho-1} & \text { if } \quad N=1, \frac{1}{2}<\rho<1, \\
1+\frac{4(3-2 \rho)}{2(2 \rho-1)} & \text { if } \quad N=2, \frac{1}{2}<\rho<1, \\
1+\frac{13-8 \rho}{4 \rho+1} & \text { if } \quad N=3, \frac{11}{6}<\rho<1 .
\end{array}\right.
$$

Studying the same problem (2) and (5), D'Abbicco [15] obtained a global existence result for $q>q(N, \rho)$, where $N \leq 5$ and $\rho \in(0,1)$, imposing suitable initial conditions.

The previous contributors give motivation to our work here. Indeed, we aim to study the effect of the inhomogeneous term $\mu$ on the large time behavior for problem (2) and (5).

Under sufficient conditions on the inhomogeneous term $\mu$ and the functions $\phi_{i} \in L_{l o c}^{1}\left(\mathbb{R}^{N}\right)$, $i=0,1$, a nonexistence result is given in the following main result.

Theorem 1. If

$$
\mu \in L^{1}\left(\mathbb{R}^{N}\right), \quad \int_{\mathbb{R}^{N}} \mu(\omega) d \omega>0, \quad \text { and } \quad \phi_{i} \geq 0, i=0,1,
$$

then problem (1) and (2) admits no global weak solution, for all $q>1$.

Remark 2. As a byproduct of Theorem 1, one deduces that the critical exponent for problem (1) and (2) is equal to $\infty$, for all $\rho \in(0,1)$.

In the next Section 2 we collect the auxiliary mathematical tools which we will need in establishing the proof of Theorem 1 (see Section 3). 


\section{Preliminaries}

We need some properties of fractional calculus to provide a definition of global weak solution to problem (1) and (2).

Fixing $\tau>0$, for given $\eta>0$ and $\xi \in C([0, \tau])$, we recall the fractional integrals:

$$
\left(I_{0}^{\eta} \xi\right)(t)=\frac{1}{\Gamma(\eta)} \int_{0}^{t}(t-\sigma)^{\eta-1} \xi(\sigma) d \sigma, \quad 0<t \leq \tau
$$

and

$$
\left(I_{\tau}^{\eta} \xi\right)(t)=\frac{1}{\Gamma(\eta)} \int_{t}^{\tau}(\sigma-t)^{\eta-1} \xi(\sigma) d \sigma, \quad 0 \leq t<\tau .
$$

From Kilbas-Srivastava-Trjillo [16], for $\eta>0$ and $\xi_{i} \in C([0, \tau]), i=1,2$, one has

$$
\int_{0}^{\tau}\left(I_{0}^{\eta} \xi_{1}\right)(t) \xi_{2}(t) d t=\int_{0}^{\tau} \xi_{1}(t)\left(I_{\tau}^{\eta} \xi_{2}\right)(t) d t
$$

Notice that (1) can be written as

$$
\phi_{t t}-\Delta \phi=\left(I_{0}^{1-\rho}|\phi(\cdot, \omega)|^{q}\right)(t)+\mu(\omega) \quad \text { in }(0, \infty) \times \mathbb{R}^{N} .
$$

Let $\Omega=[0, \infty) \times \mathbb{R}^{N}$. For $\tau>0$, let $\Omega_{\tau}=[0, \tau] \times \mathbb{R}^{N}$ and $V_{\tau}$ be the test function space defined by

$$
V_{\tau}=\left\{\vartheta \in C^{2}\left(\Omega_{\tau}\right): \operatorname{supp}_{\omega} \vartheta \subset \subset \mathbb{R}^{N}, \vartheta(\tau, \omega)=\vartheta_{t}(\tau, \omega)=0\right\} .
$$

Combining (6) and (7), we may define global weak solutions to (1) and (2) as follows.

Definition 1. We say that $\phi$ is a global weak solution to problem (1) and (2), if $\phi \in L_{l o c}^{q}(\Omega)$ and

$$
\begin{aligned}
& \int_{\Omega_{\tau}}|\phi|{ }^{q} I_{\tau}^{1-\rho} \vartheta d \omega d t+\int_{\Omega_{\tau}} \mu(\omega) \vartheta d \omega d t+\int_{\mathbb{R}^{N}} \phi_{0}(\omega)\left(\vartheta(0, \omega)-\vartheta_{t}(0, \omega)\right) d \omega+\int_{\mathbb{R}^{N}} \phi_{1}(\omega) \vartheta(0, \omega) d \omega \\
& =\int_{\Omega_{\tau}} \phi \vartheta_{t t} d \omega d t-\int_{\Omega_{\tau}} \phi \vartheta_{t} d \omega d t-\int_{\Omega_{\tau}} \phi \Delta \vartheta d \omega d t
\end{aligned}
$$

for all $\tau>0$ and $\vartheta \in V_{\tau}$.

One of the tools of this study is the cut-off functions method. Here, we introduce a cut-off function $\kappa \in C^{\infty}([0, \infty))$ such that

$$
0 \leq \kappa \leq 1 ; \quad \kappa \equiv 1 \text { in }[0,1] ; \quad \kappa \equiv 0 \text { in }[2, \infty) .
$$

In addition, for the sake of simplicity, fixing $\tau, L>0$, we introduce the functions:

$$
a_{\tau}(t)=\tau^{-\delta}(\tau-t)^{\delta}, \quad 0 \leq t \leq \tau
$$

and

$$
b_{L}(\omega)=\kappa\left(\frac{|\omega|^{2}}{L^{2}}\right)^{\varsigma}, \quad \omega \in \mathbb{R}^{N},
$$

where $\delta, \varsigma \geq 2$ are constants. Based on these functions, we construct a two-variable function $\vartheta$ as follows:

$$
\vartheta(t, \omega)=\vartheta_{\tau, L}(t, \omega)=a_{\tau}(t) b_{L}(\omega), \quad(t, \omega) \in \Omega_{\tau} .
$$

Such a function $\vartheta$ satisfies the following result, whose proof is immediate. 
Lemma 1. For all $\tau, L>0$, the function $\vartheta$ defined by (10) belongs to $V_{\tau}$, where $V_{\tau}$ is the test function space defined by (8).

We establish the following lemmata about the properties of function $b_{L}$.

Lemma 2. There exists a constant $C>0$ such that

$$
\int_{\mathbb{R}^{N}} b_{L}(\omega) d \omega=C L^{N}, \quad L>0
$$

Proof. Performing integration over the definition of $b_{L}$, we have

$$
\int_{\mathbb{R}^{N}} b_{L}(\omega) d \omega=\int_{\mathbb{R}^{N}} \kappa\left(\frac{|\omega|^{2}}{L^{2}}\right)^{\varsigma} d \omega
$$

Acting with the definition of the cut-off function $\kappa$, we obtain

$$
\int_{\mathbb{R}^{N}} b_{L}(\omega) d \omega=\int_{0<|w|<\sqrt{2} L} \kappa\left(\frac{|\omega|^{2}}{L^{2}}\right)^{\varsigma} d \omega
$$

Finally, taking $z=L^{-1} \omega$, we deduce that

$$
\int_{\mathbb{R}^{N}} b_{L}(\omega) d \omega=L^{N} \int_{0<|z|<\sqrt{2}} \kappa\left(|z|^{2}\right)^{\varsigma} d z,
$$

which proves the desired result, where $C$ is a constant equal to the integral in the right-hand side of the above equation.

Lemma 3. Let $\varsigma>\frac{2 q}{q-1}$. There exists a constant $C>0$ such that

$$
\int_{\mathbb{R}^{N}}\left|b_{L}(\omega)\right|^{\frac{-1}{q-1}}\left|\Delta b_{L}(\omega)\right|^{\frac{q}{q-1}} d \omega \leq C L^{\frac{-2 q}{q-1}+N}
$$

Proof. Using the similar arguments as in the proof of Lemma 2 above, that is, performing integration over the definition of $b_{L}$, and acting with the properties of the cut-off function $\kappa$, we obtain

$$
\int_{\mathbb{R}^{N}}\left|b_{L}(\omega)\right|^{\frac{-1}{q-1}}\left|\Delta b_{L}(\omega)\right|^{\frac{q}{q-1}} d \omega=\int_{L<|\omega|<\sqrt{2} L} \kappa\left(\frac{|\omega|^{2}}{L^{2}}\right)^{\frac{-\zeta}{q-1}}\left|\Delta \kappa\left(\frac{|\omega|^{2}}{L^{2}}\right)^{\varsigma}\right|^{\frac{q}{q-1}} d \omega .
$$

In order to manipulate the above equation, to get the aimed result, we perform some elementary calculations and have

$$
\Delta \kappa\left(\frac{|\omega|^{2}}{L^{2}}\right)^{\varsigma}=2 L^{-2} \zeta \mathcal{K}\left(\frac{|\omega|^{2}}{L^{2}}\right)^{\zeta-2} \psi(\omega), \quad L<|\omega|<\sqrt{2} L
$$

where

$$
\begin{aligned}
\psi(\omega)= & \kappa\left(\frac{|\omega|^{2}}{L^{2}}\right) \kappa^{\prime}\left(\frac{|\omega|^{2}}{L^{2}}\right)+2 L^{-2}|\omega|^{2}(\varsigma-1) \kappa^{\prime}\left(\frac{|\omega|^{2}}{L^{2}}\right)^{2}+2 L^{-2}|\omega|^{2} \kappa\left(\frac{|\omega|^{2}}{L^{2}}\right) \kappa^{\prime \prime}\left(\frac{|\omega|^{2}}{L^{2}}\right) \\
& +(N-1) \kappa\left(\frac{|\omega|^{2}}{L^{2}}\right) \kappa^{\prime}\left(\frac{|\omega|^{2}}{L^{2}}\right) .
\end{aligned}
$$

Invoking the properties of the cut-off function $\kappa$, we deduce that there exists a constant $C_{\kappa}>0$ such that

$$
2 \varsigma|\psi(\omega)| \leq C_{\kappa}, \quad L<|\omega|<\sqrt{2} L
$$


which yields to

$$
\left|\Delta \kappa\left(\frac{|\omega|^{2}}{L^{2}}\right)^{\varsigma}\right| \leq C_{\kappa} L^{-2} \mathcal{K}\left(\frac{|\omega|^{2}}{L^{2}}\right)^{\varsigma-2}, \quad L<|\omega|<\sqrt{2} L .
$$

Therefore, we deduce that

$$
\kappa\left(\frac{|\omega|^{2}}{L^{2}}\right)^{\frac{-\zeta}{q-1}}\left|\Delta \kappa\left(\frac{|\omega|^{2}}{L^{2}}\right)^{\varsigma}\right|^{\frac{q}{q-1}} \leq C_{\kappa}^{\frac{q}{q-1}} L^{\frac{-2 q}{q-1}} \kappa\left(\frac{|\omega|^{2}}{L^{2}}\right)^{\varsigma-\frac{2 q}{q-1}}, \quad L<|\omega|<\sqrt{2} L
$$

and hence

$$
\int_{\mathbb{R}^{N}}\left|b_{L}(\omega)\right|^{\frac{-1}{q-1}}\left|\Delta b_{L}(\omega)\right|^{\frac{q}{q-1}} d \omega \leq C_{\kappa}^{\frac{q}{q-1}} L^{\frac{-2 q}{q-1}} \int_{L<|\omega|<\sqrt{2} L} \kappa\left(\frac{|\omega|^{2}}{L^{2}}\right)^{\zeta-\frac{2 q}{q-1}} d \omega .
$$

Taking $z=L^{-1} \omega$, it holds that

$$
\int_{\mathbb{R}^{N}}\left|b_{L}(\omega)\right|^{\frac{-1}{q-1}}\left|\Delta b_{L}(\omega)\right|^{\frac{q}{q-1}} d \omega \leq C_{\kappa}^{\frac{q}{q-1}} L^{\frac{-2 q}{q-1}+N} \int_{1<|z|<\sqrt{2}} \kappa\left(|z|^{2}\right)^{\varsigma-\frac{2 q}{q-1}} d z
$$

Since $\varsigma>\frac{2 q}{q-1}$, we derive easily the desired estimate.

And now, we have to consider the function $a_{\tau}$. The authors in a previous paper (see the proof of Theorem 1, p. 9, Equation (24), of [17]) obtained the following result:

Lemma 4. For all $0 \leq t<\tau$, we have

$$
\left(I_{\tau}^{1-\rho} a_{\tau}\right)(t)=\frac{\Gamma(\delta+1)}{\Gamma(\delta+2-\rho)} \tau^{-\delta}(\tau-t)^{\delta+1-\rho} .
$$

Here, we prove the following additional results:

Lemma 5. Let $\delta>\frac{2 q+1-\rho}{q-1}$. There exists a constant $C>0$ such that

$$
\int_{0}^{\tau}\left|\left(I_{\tau}^{1-\rho} a_{\tau}\right)(t)\right|^{\frac{-1}{q-1}}\left|a_{\tau}^{\prime \prime}(t)\right|^{\frac{q}{q-1}} d t=C \tau^{\frac{\rho-q-2}{q-1}} .
$$

Proof. By the definition of the function $a_{\tau}$, performing double derivation, we have

$$
a_{\tau}^{\prime \prime}(t)=\delta(\delta-1) \tau^{-\delta}(\tau-t)^{\delta-2}, \quad 0 \leq t \leq \tau .
$$

Hence, using Lemma 4, we obtain

$$
\left|\left(I_{\tau}^{1-\rho} a_{\tau}\right)(t)\right|^{\frac{-1}{q-1}}\left|a_{\tau}^{\prime \prime}(t)\right|^{\frac{q}{q-1}}=C_{\delta, \rho} \tau^{-\delta}(\tau-t)^{\delta-\frac{2 q+1-\rho}{q-1}}, \quad 0 \leq t \leq \tau,
$$

where

$$
C_{\delta, \rho}=[\delta(\delta-1)]^{\frac{q}{q-1}}\left[\frac{\Gamma(\delta+1)}{\Gamma(\delta+2-\rho)}\right]^{\frac{-1}{q-1}} .
$$

Performing integration over $[0, \tau]$, the above equation gives us

$$
\begin{aligned}
\int_{0}^{\tau}\left|\left(I_{\tau}^{1-\rho} a_{\tau}\right)(t)\right|^{\frac{-1}{q-1}}\left|a_{\tau}^{\prime \prime}(t)\right|^{\frac{q}{q-1}} d t & =C_{\delta, \rho} \tau^{-\delta} \int_{0}^{\tau}(\tau-t)^{\delta-\frac{2 q+1-\rho}{q-1}} d t \\
& =C_{\delta, \rho} \tau^{-\frac{2 q+1-\rho}{q-1}} \int_{0}^{\tau}\left(1-\frac{t}{\tau}\right)^{\delta-\frac{2 q+1-\rho}{q-1}} d t
\end{aligned}
$$


Taking $s=\frac{t}{\tau}$, it holds that

$$
\begin{aligned}
\int_{0}^{\tau}\left|\left(I_{\tau}^{1-\rho} a_{\tau}\right)(t)\right|^{\frac{-1}{q-1}}\left|a_{\tau}^{\prime \prime}(t)\right|^{\frac{q}{q-1}} d t & =C_{\delta, \rho} \tau^{1-\frac{2 q+1-\rho}{q-1}} \int_{0}^{1}(1-s)^{\delta-\frac{2 q+1-\rho}{q-1}} d s \\
& =C_{\delta, \rho} \tau^{\frac{\rho-q-2}{q-1}} \int_{0}^{1}(1-s)^{\delta-\frac{2 q+1-\rho}{q-1}} d s
\end{aligned}
$$

which leads to the desired estimate, as $\delta>\frac{2 q+1-\rho}{q-1}$.

Lemma 6. Let $\delta>\frac{q+1-\rho}{q-1}$. There exists a constant $C>0$ such that

$$
\int_{0}^{\tau}\left|\left(I_{\tau}^{1-\rho} a_{\tau}\right)(t)\right|^{\frac{-1}{q-1}}\left|a_{\tau}^{\prime}(t)\right|^{\frac{q}{q-1}} d t=C \tau^{\frac{\rho-2}{q-1}} .
$$

Proof. Starting from the definition of $a_{\tau}$ and performing derivation, we have

$$
a_{\tau}^{\prime}(t)=-\delta \tau^{-\delta}(\tau-t)^{\delta-1}, \quad 0 \leq t \leq \tau .
$$

Next, an application of Lemma 4 leads us to

$$
\left|\left(I_{\tau}^{1-\rho} a_{\tau}\right)(t)\right|^{\frac{-1}{q-1}}\left|a_{\tau}^{\prime}(t)\right|^{\frac{q}{q-1}}=\delta^{\frac{q}{q-1}} \tau^{-\delta}(\tau-t)^{\delta-\frac{q+1-\rho}{q-1}}, \quad 0 \leq t \leq \tau .
$$

We integrate over $[0, \tau]$ to get

$$
\begin{aligned}
\int_{0}^{\tau}\left|\left(I_{\tau}^{1-\rho} a_{\tau}\right)(t)\right|^{\frac{-1}{q-1}}\left|a_{\tau}^{\prime}(t)\right|^{\frac{q}{q-1}} d t & =\delta^{\frac{q}{q-1}} \tau^{-\delta} \int_{0}^{\tau}(\tau-t)^{\delta-\frac{q+1-\rho}{q-1}} d t \\
& =\delta^{\frac{q}{q-1}} \tau^{-\frac{q+1-\rho}{q-1}} \int_{0}^{\tau}\left(1-\frac{t}{\tau}\right)^{\delta-\frac{q+1-\rho}{q-1}} d t
\end{aligned}
$$

Taking $s=\frac{t}{\tau}$, it holds that

$$
\begin{aligned}
\int_{0}^{\tau}\left|\left(I_{\tau}^{1-\rho} a_{\tau}\right)(t)\right|^{\frac{-1}{q-1}}\left|a_{\tau}^{\prime}(t)\right|^{\frac{q}{q-1}} d t & =\delta^{\frac{q}{q-1}} \tau^{1-\frac{q+1-\rho}{q-1}} \int_{0}^{1}(1-s)^{\delta-\frac{q+1-\rho}{q-1}} d s \\
& =\delta^{\frac{q}{q-1}} \tau^{\frac{\rho-2}{q-1}} \int_{0}^{1}(1-s)^{\delta-\frac{q+1-\rho}{q-1}} d s
\end{aligned}
$$

which yields the desired result (recall that $\delta>\frac{q+1-\rho}{q-1}$ ).

Lemma 7. Let $\delta>\frac{1-\rho}{q-1}$. There exists a constant $C>0$ such that

$$
\int_{0}^{\tau}\left|\left(I_{\tau}^{1-\rho} a_{\tau}\right)(t)\right|^{\frac{-1}{q-1}} a_{\tau}(t)^{\frac{q}{q-1}} d t=C \tau^{\frac{q-2+\rho}{q-1}}
$$

Proof. Combining the definition of the function $a_{\tau}$ with the estimate in Lemma 4, we obtain

$$
\left|\left(I_{\tau}^{1-\rho} a_{\tau}\right)(t)\right|^{\frac{-1}{q-1}} a_{\tau}(t)^{\frac{q}{q-1}}=C_{\delta, \rho} \tau^{-\delta}(\tau-t)^{\delta-\frac{1-\rho}{q-1}}, \quad 0 \leq t<\tau,
$$

where $C_{\delta, \rho}=\left[\frac{\Gamma(\delta+1)}{\Gamma(\delta+2-\rho)}\right]^{\frac{-1}{q-1}}$.

Integrating over the interval $[0, \tau]$, we deduce that 


$$
\int_{0}^{\tau}\left|\left(I_{\tau}^{1-\rho} a_{\tau}\right)(t)\right|^{\frac{-1}{q-1}} a_{\tau}(t)^{\frac{q}{q-1}} d t=C_{\delta, \rho} \tau^{\frac{\rho-1}{q-1}} \int_{0}^{\tau}\left(1-\frac{t}{\tau}\right)^{\delta-\frac{1-\rho}{q-1}} d t .
$$

Taking $s=\frac{t}{\tau}$, it follows easily that

$$
\int_{0}^{\tau}\left|\left(I_{\tau}^{1-\rho} a_{\tau}\right)(t)\right|^{\frac{-1}{q-1}} a_{\tau}(t)^{\frac{q}{q-1}} d t=C_{\delta, \rho} \tau^{\frac{q-2+\rho}{q-1}} \int_{0}^{1}(1-s)^{\delta-\frac{1-\rho}{q-1}} d s .
$$

Since $\delta>\frac{1-\rho}{q-1}$, we have established our goal here.

The last lemma of this section provides an immediate property of the function $a_{\tau}$.

Lemma 8. There exists a constant $C>0$ such that

$$
\int_{0}^{\tau} a_{\tau}(t) d t=C \tau
$$

\section{Proof of Theorem 1}

For the sake of notational simplicity, in the sequel we denote by $C$ any positive constant that is independent on $\tau$. So, we give the complete proof of Theorem 1 .

Proof. We argue by contradiction. So, we suppose that $\phi \in L_{l o c}^{q}(\Omega)$ is a global weak solution to problem (1) and (2) (in the sense of Definition 1). For $\tau>0$, we define the set

$$
W_{\tau}=\left\{\vartheta \in V_{\tau}: I_{\tau}^{1-\rho} \vartheta \geq 0, I_{\tau}^{1-\rho} \vartheta \not \equiv 0\right\} .
$$

Then, by (9), for all $\tau>0$ and $\vartheta \in W_{\tau}$, we have

$$
\begin{aligned}
& \int_{\Omega_{\tau}}|\phi|^{q} I_{\tau}^{1-\rho} \vartheta d \omega d t+\int_{\Omega_{\tau}} \mu(\omega) \vartheta d \omega d t+\int_{\mathbb{R}^{N}} \phi_{0}(\omega)\left(\vartheta(0, \omega)-\vartheta_{t}(0, \omega)\right) d \omega+\int_{\mathbb{R}^{N}} \phi_{1}(\omega) \vartheta(0, \omega) d \omega \\
& \leq \int_{\Omega_{\tau}}|\phi|\left|\vartheta_{t t}\right| d \omega d t+\int_{\Omega_{\tau}}|\phi|\left|\vartheta_{t}\right| d \omega d t+\int_{\Omega_{\tau}}|\phi||\Delta \vartheta| d \omega d t .
\end{aligned}
$$

On the other hand, we use the $\epsilon$-Young inequality with $\epsilon=\frac{1}{3}$, to compute the following three estimates:

$$
\begin{aligned}
\int_{\Omega_{\tau}}|\phi|\left|\vartheta_{t t}\right| d \omega d t & =\int_{\Omega_{\tau}}\left[|\phi|\left(I_{\tau}^{1-\rho} \vartheta\right)^{\frac{1}{q}}\right]\left[\left(I_{\tau}^{1-\rho} \vartheta\right)^{\frac{-1}{q}}\left|\vartheta_{t t}\right|\right] d \omega d t \\
& \leq \frac{1}{3} \int_{\Omega_{\tau}}|\phi|^{q} I_{\tau}^{1-\rho} \vartheta d \omega d t+C \int_{\Omega_{\tau}}\left(I_{\tau}^{1-\rho} \vartheta\right)^{\frac{-1}{q-1}}\left|\vartheta_{t t}\right|^{\frac{q}{q-1}} d \omega d t
\end{aligned}
$$

and

$$
\int_{\Omega_{\tau}}|\phi|\left|\vartheta_{t}\right| d \omega d t \leq \frac{1}{3} \int_{\Omega_{\tau}}|\phi|^{q} I_{\tau}^{1-\rho} \vartheta d \omega d t+C \int_{\Omega_{\tau}}\left(I_{\tau}^{1-\rho} \vartheta\right)^{\frac{-1}{q-1}}\left|\vartheta_{t}\right|^{\frac{q}{q-1}} d \omega d t
$$

and

$$
\int_{\Omega_{\tau}}|\phi||\Delta \vartheta| d \omega d t \leq \frac{1}{3} \int_{\Omega_{\tau}}|\phi|^{q} I_{\tau}^{1-\rho} \vartheta d \omega d t+C \int_{\Omega_{\tau}}\left(I_{\tau}^{1-\rho} \vartheta\right)^{\frac{-1}{q-1}}|\Delta \vartheta|^{\frac{q}{q-1}} d \omega d t
$$

Using (12)-(14) in (11), we get

$$
\begin{aligned}
& \int_{\Omega_{\tau}}|\phi|^{q} I_{\tau}^{1-\rho} \vartheta d \omega d t+\int_{\Omega_{\tau}} \mu(\omega) \vartheta d \omega d t+\int_{\mathbb{R}^{N}} \phi_{0}(\omega)(\vartheta(0, \omega)-\vartheta t(0, \omega)) d \omega+\int_{\mathbb{R}^{N}} \phi_{1}(\omega) \vartheta(0, \omega) d \omega \\
& \leq \int_{\Omega_{\tau}}|\phi|^{q} I_{\tau}^{1-\rho} \vartheta d \omega d t \\
& \quad+C\left(\int_{\Omega_{\tau}}\left(I_{\tau}^{1-\rho} \vartheta\right)^{\frac{-1}{q-1}}\left|\vartheta_{t t}\right|^{\frac{q}{q-1}} d \omega d t+\int_{\Omega_{\tau}}\left(I_{\tau}^{1-\rho} \vartheta\right)^{\frac{-1}{q-1}}\left|\vartheta_{t}\right|^{\frac{q}{q-1}} d \omega d t+\int_{\Omega_{\tau}}\left(I_{\tau}^{1-\rho} \vartheta\right)^{\frac{-1}{q-1}}|\Delta \vartheta|^{\frac{q}{q-1}} d \omega d t\right) .
\end{aligned}
$$


Consequently, we have

$$
\begin{aligned}
& \int_{\Omega_{\tau}} \mu(\omega) \vartheta d \omega d t+\int_{\mathbb{R}^{N}} \phi_{0}(\omega)\left(\vartheta(0, \omega)-\vartheta_{t}(0, \omega)\right) d \omega+\int_{\mathbb{R}^{N}} \phi_{1}(\omega) \vartheta(0, \omega) d \omega \\
& \leq C\left(\int_{\Omega_{\tau}}\left(I_{\tau}^{1-\rho} \vartheta\right)^{\frac{-1}{q-1}}\left|\vartheta_{t t}\right|^{\frac{q}{q-1}} d \omega d t+\int_{\Omega_{\tau}}\left(I_{\tau}^{1-\rho} \vartheta\right)^{\frac{-1}{q-1}}\left|\vartheta_{t}\right|^{\frac{q}{q-1}} d \omega d t+\int_{\Omega_{\tau}}\left(I_{\tau}^{1-\rho} \vartheta\right)^{\frac{-1}{q-1}}|\Delta \vartheta|^{\frac{q}{q-1}} d \omega d t\right) .
\end{aligned}
$$

We recall that the inequality (15) holds for all $\tau>0$ and $\vartheta \in W_{\tau}$. On the other hand, by Lemma 1 , we know that for all $\tau, L>0$, the function $\vartheta$ defined by (10) belongs to $V_{\tau}$. Moreover, we point out that

$$
\left.I_{\tau}^{1-\rho} \vartheta \geq 0 \text { and } I_{\mathcal{\tau}}^{1-\rho} \vartheta \not \equiv 0 \text { (by Lemma } 4\right)
$$

So, for all $\tau, L>0$, the function $\vartheta$ (defined by (10)) belongs to $W_{\tau}$. It follows that such a function $\vartheta$ is suitable as test function in (15), and (obviously) $\tau, L>0$. Moreover, we take $\delta$ and $\varsigma$ greater than two threshold values, that is

$$
\delta>\frac{2 q+1-\rho}{q-1}
$$

and

$$
\varsigma>\frac{2 q}{q-1}
$$

Now, we combine such "ingredients" to obtain suitable estimates of the three terms in the right-hand side of (15).

- Estimate of $\int_{\Omega_{\tau}}\left(I_{\tau}^{1-\rho} \vartheta\right)^{\frac{-1}{q-1}}\left|\vartheta_{t t}\right|^{\frac{q}{q-1}} d \omega d t$

By (10), we obtain

$$
\int_{\Omega_{\tau}}\left(I_{\tau}^{1-\rho} \vartheta\right)^{\frac{-1}{q-1}}\left|\vartheta_{t t}\right|^{\frac{q}{q-1}} d \omega d t=\left(\int_{\mathbb{R}^{N}} b_{L}(\omega) d \omega\right)\left(\int_{0}^{\tau}\left|\left(I_{\tau}^{1-\rho} a_{\tau}\right)(t)\right|^{\frac{-1}{q-1}}\left|a_{\tau}^{\prime \prime}(t)\right|^{\frac{q}{q-1}} d t\right) .
$$

By (16), Lemmata 2 and 5 give us

$$
\int_{\Omega_{\tau}}\left(I_{\tau}^{1-\rho} \vartheta\right)^{\frac{-1}{q-1}}\left|\vartheta_{t t}\right|^{\frac{q}{q-1}} d \omega d t=C L^{N} \tau^{\frac{\rho-q-2}{q-1}}
$$

- Estimate of $\int_{\Omega_{\tau}}\left(I_{\tau}^{1-\rho} \vartheta\right)^{\frac{-1}{q-1}}\left|\vartheta_{t}\right|^{\frac{q}{q-1}} d \omega d t$

Again starting by (10), we have

$$
\int_{\Omega_{\tau}}\left(I_{\tau}^{1-\rho} \vartheta\right)^{\frac{-1}{q-1}}\left|\vartheta_{t}\right|^{\frac{q}{q-1}} d \omega d t=\left(\int_{\mathbb{R}^{N}} b_{L}(\omega) d \omega\right)\left(\int_{0}^{\tau}\left|\left(I_{\tau}^{1-\rho} a_{\tau}\right)(t)\right|^{\frac{-1}{q-1}} \mid a_{\tau}^{\prime}(t)^{\frac{q}{q-1}} d t\right) .
$$

Then, by (16), Lemmata 2 and 6 lead to

$$
\int_{\Omega_{\tau}}\left(I_{\tau}^{1-\rho} \vartheta\right)^{\frac{-1}{q-1}}\left|\vartheta_{t}\right|^{\frac{q}{q-1}} d \omega d t=C L^{N} \tau^{\frac{\rho-2}{q-1}}
$$

- Estimate of $\int_{\Omega_{\tau}}\left(I_{\tau}^{1-\rho} \vartheta\right)^{\frac{-1}{q-1}}|\Delta \vartheta|^{\frac{q}{q-1}} d \omega d t$

By (10), it holds that

$$
\int_{\Omega_{\tau}}\left(I_{\tau}^{1-\rho} \vartheta\right)^{\frac{-1}{q-1}}|\Delta \vartheta|^{\frac{q}{q-1}} d \omega d t=\left(\int_{0}^{\tau} a_{\tau}(t)^{\frac{q}{q-1}}\left(I_{\tau}^{1-\rho} a_{\tau}\right)(t)^{\frac{-1}{q-1}} d t\right)\left(\int_{\mathbb{R}^{N}}\left|b_{L}(\omega)\right|^{\frac{-1}{q-1}} \mid \Delta b_{L}(\omega)^{\frac{q}{q-1}} d \omega\right) .
$$


So, Lemmata 3 and 7, through to (16) and (17), provide the estimate

$$
\int_{\Omega_{\tau}}\left(I_{\tau}^{1-\rho} \vartheta\right)^{\frac{-1}{q-1}}|\Delta \vartheta|^{\frac{q}{q-1}} d \omega d t \leq C L^{\frac{-2 q}{q-1}+N} \tau^{\frac{q-2+\rho}{q-1}} .
$$

The combinated effects of (18)-(20) in (15) lead to the inequality

$$
\begin{aligned}
& \int_{\Omega_{\tau}} \mu(\omega) \vartheta d \omega d t+\int_{\mathbb{R}^{N}} \phi_{0}(\omega)\left(\vartheta(0, \omega)-\vartheta_{t}(0, \omega)\right) d \omega+\int_{\mathbb{R}^{N}} \phi_{1}(\omega) \vartheta(0, \omega) d \omega \\
& \leq C\left(L^{N} \tau^{\frac{\rho-q-2}{q-1}}+L^{N} \tau^{\frac{\rho-2}{q-1}}+L^{\frac{-2 q}{q-1}+N} \tau^{\frac{q-2+\rho}{q-1}}\right) .
\end{aligned}
$$

Now, we manipulate the left-hand side of (21). Indeed, we know that

$$
\int_{\Omega_{\tau}} \mu(\omega) \vartheta d \omega d t=\left(\int_{0}^{\tau} a_{\tau}(t) d t\right)\left(\int_{\mathbb{R}^{N}} \mu(\omega) b_{L}(\omega) d \omega\right) \quad \text { (by (10)). }
$$

By the definition of the function $b_{L}$, and using Lemma 8 , we obtain

$$
\int_{\Omega_{\tau}} \mu(\omega) \vartheta d \omega d t=C \tau \int_{\mathbb{R}^{N}} \mu(\omega) \kappa\left(\frac{|\omega|^{2}}{L^{2}}\right)^{\varsigma} d \omega
$$

Notice that since $\mu \in L^{1}\left(\mathbb{R}^{N}\right)$, by the properties of the cut-off function $\kappa$, and using the dominated convergence theorem, it holds that

$$
\lim _{L \rightarrow \infty} \int_{\mathbb{R}^{N}} \mu(\omega) \kappa\left(\frac{|\omega|^{2}}{L^{2}}\right)^{\varsigma} d \omega=\int_{\mathbb{R}^{N}} \mu(\omega) d \omega
$$

Since $\int_{\mathbb{R}^{N}} \mu(\omega) d \omega>0$, we deduce that there exists a constant $L_{0}>0$ such that

$$
\int_{\mathbb{R}^{N}} \mu(\omega) \mathcal{K}\left(\frac{|\omega|^{2}}{L^{2}}\right)^{\varsigma} d \omega \geq \frac{1}{2} \int_{\mathbb{R}^{N}} \mu(\omega) d \omega, \quad L>L_{0}
$$

Therefore, combining (22) and (23), we obtain

$$
\int_{\Omega_{\tau}} \mu(\omega) \vartheta d \omega d t \geq C \tau \int_{\mathbb{R}^{N}} \mu(\omega) d \omega, \quad L>L_{0}
$$

On the other hand, by (10) we have

$$
\int_{\mathbb{R}^{N}} \phi_{0}(\omega)\left(\vartheta(0, \omega)-\vartheta_{t}(0, \omega)\right) d \omega+\int_{\mathbb{R}^{N}} \phi_{1}(\omega) \vartheta(0, \omega) d \omega=\int_{\mathbb{R}^{N}} b_{L}(\omega)\left[\left(1+\delta \tau^{-1}\right) \phi_{0}(\omega)+\phi_{1}(\omega)\right] d \omega .
$$

Since $b_{L}, \phi_{i} \geq 0, i=0,1$, we deduce that

$$
\int_{\mathbb{R}^{N}} \phi_{0}(\omega)\left(\vartheta(0, \omega)-\vartheta_{t}(0, \omega)\right) d \omega+\int_{\mathbb{R}^{N}} \phi_{1}(\omega) \vartheta(0, \omega) d \omega \geq 0 .
$$

Acting on the left-hand side of (21), it follows from (24) and (25) that

$$
\int_{\mathbb{R}^{N}} \mu(\omega) d \omega \leq C\left(L^{N} \tau^{\frac{\rho-2 q-1}{q-1}}+L^{N} \tau^{\frac{\rho-q-1}{q-1}}+L^{\frac{-2 q}{q-1}+N} \tau^{\frac{\rho-1}{q-1}}\right), \quad L>L_{0}, \tau>0 .
$$

Notice that

$$
\frac{\rho-2 q-1}{q-1}<0, \quad \frac{\rho-q-1}{q-1}<0, \quad \frac{\rho-1}{q-1}<0
$$


Hence, fixing $L>L_{0}$ and passing to the limit as $\tau \rightarrow \infty$ in (26), we obtain

$$
\int_{\mathbb{R}^{N}} \mu(\omega) d \omega \leq 0,
$$

which contradicts the fact that $\int_{\mathbb{R}^{N}} \mu(\omega) d \omega>0$. We conclude that problem (1) and (2) admits no global weak solution.

\section{Conclusions}

The presented results confirm usefulness and simplicity of the test function method in analyzing different forms of wave equations. As a consequence of this approach in the previous sections, it is possible to see that the inhomogeneous damped wave equation with nonlinear memory (1) and (2) admits no global weak solution. In particular, we point out the absence of critical growth exponent $q>1$ for the nonlinear memory, in proving such a result.

This topic may be significant for the study of the controllability of solutions to certain nonlinear models of physics systems, together with the symmetry analysis. We have already mentioned in Section 1 above, a possible relationship between the boundary conditions, and the properties and behavior of a physical system. Here we imposed a Cauchy condition, but it will be interesting to know how Neumann, Robin and mixed boundary conditions affect the analysis of the wave type problem (1) and (2).

Author Contributions: Investigation, all authors; Writing—original draft, all authors. All authors have read and agreed to the published version of the manuscript.

Funding: The first author is supported by Researchers Supporting Project number (RSP-2020/57), King Saud University, Riyadh, Saudi Arabia.

Acknowledgments: The authors are very grateful to the referees for the valuable comments and suggestions.

Conflicts of Interest: The authors declare no conflict of interest.

\section{References}

1. Love, A.E.H. A Treatise on the Mathematical Theory of Elasticity; Dover Publications: New York, NY, USA, 1944.

2. Woodhouse, J.H. Surface waves in a laterally varying layered structure. Geophys. J. R. Astron. Soc. 1974, 37, 461-490. [CrossRef]

3. Chapman, C.H.; Woodhouse, J.H. Symmetry of the wave equation and excitation of body waves. Geophys. J. R. Astron. Soc. 1981, 65, 111-182. [CrossRef]

4. Bluman, G.; Cheviakov, A.F. Nonlocally related systems, linearization and nonlocalsymmetries for the nonlinear wave equation. J. Math. Anal. Appl. 2007, 333, 93-111. [CrossRef]

5. Taylor, N.W.; Kidder, L.E.; Teukolsky, S.A. Spectral methods for the wave equation in second-order form. Phys. Rev. D 2010, 82, 024037. [CrossRef]

6. Palacz, M. Spectral methods for modelling of wave propagation in structures in terms of damage detection-A review. Appl. Sci. 2018, 8, 1124. [CrossRef]

7. Todorova, G.; Yordanov, B. Critical exponent for a nonlinear wave equation with damping. J. Differ. Equ. 2001, 174, 464-489. [CrossRef]

8. Fujita, H. On the blowing-up of solutions to the Cauchy problem for $u_{t}=\Delta u+u^{1+\alpha}$. J. Fac. Sci. Univ. Tokyo Sect. IA Math. 1990, 13, 109-124.

9. Kirane, M.; Qafsaoui, M. Fujita's exponent for a semilinear wave equation with linear damping. Adv. Nonlinear Stud. 2002, 2, 41-49. [CrossRef]

10. Zhang, Q.S. A blow-up result for a nonlinear wave equation with damping: The critical case. C. R. Acad. Sci. Paris Ser. I 2001, 333, 109-114. [CrossRef]

11. Jleli, M.; Samet, B. New critical behaviors for semilinear wave equations and systems with linear damping terms. Math. Methods Appl. Sci. 2020, 1-11. [CrossRef]

12. Zhang, Q.S. A new critical phenomenon for semilinear parabolic problems. J. Math. Anal. Appl. 1998, 219, 125-139. [CrossRef] 
13. Zhang, Q.S. A new critical behavior for nonlinear wave equations. J. Comput. Anal. Appl. 2000, 2, 277-292.

14. Fino, A.Z. Critical exponent for damped wave equations with nonlinear memory. Nonlinear Anal. 2011, 74, 5495-5505. [CrossRef]

15. D'Abbicco, M. The influence of a nonlinear memory on the damped wave equation. Nonlinear Anal. 2014, 95, 130-145. [CrossRef]

16. Kilbas, A.; Srivastava, H.; Trujillo, J. Theory and Applications of Fractional Differential Equations; Math. Studies; North-Holland: New York, NY, USA, 2006.

17. Jleli, M.; Samet, B.; Vetro, C. On a fractional in time nonlinear Schrödinger equation with dispersion parameter and absorption coefficient. Symmetry 2020, 12, 1197. [CrossRef]

(C) 2020 by the authors. Licensee MDPI, Basel, Switzerland. This article is an open access article distributed under the terms and conditions of the Creative Commons Attribution (CC BY) license (http://creativecommons.org/licenses/by/4.0/). 